\title{
Correction to: Classification of good visual acuity over time in patients with branch retinal vein occlusion with macular edema using support vector machine
}

\author{
Yoshitsugu Matsui ${ }^{1}\left[\right.$ [Dazuya Imamura ${ }^{2} \cdot$ Mihiro Ooka $^{2} \cdot$ Shinichiro Chujo $^{1} \cdot$ Yoko Mase $^{1} \cdot$ Hisashi Matsubara ${ }^{1}$. \\ Hiroharu Kawanaka ${ }^{2} \cdot$ Mineo Kondo $^{1}$
}

Published online: 17 January 2022

(c) Springer-Verlag GmbH Germany, part of Springer Nature 2022

\section{Correction to: Graefe's Archive for Clinical and Experimental Ophthalmology https://doi.org/10.1007/s00417-021-05455-y}

In the published version of this article, the Key messages details are incorrect.

The correct Key messages details are shown below:

\section{Key messages}

- It is currently an unsolved problem to predict the specific clinical outcome of individual patients before continuing treatment for macular edema associated with BRVO.

- A classifier created with handcrafted features-based support vector machine (SVM) classified patients with BRVO into two groups, one with good visual outcomes and the other with poor visual outcomes.

- $\quad$ The accuracy of the classifier was 0.806 .

- The patients' clinical information and the morphology of the outer retinal layer in OCT images within 2 degrees of the center of the macula at the first resolution of the ME were useful information for the classification of different prognosis of BCVA over time during continued anti-VEGF treatment.

This is being corrected in this publication.

Publisher's note Springer Nature remains neutral with regard to jurisdictional claims in published maps and institutional affiliations.

The original article can be found online at https://doi.org/10.1007/ s00417-021-05455-y.

Yoshitsugu Matsui

footboyslim366@gmail.com

1 Department of Ophthalmology, Mie University Graduate, School of Medicine, 2-174 Edobashi, Tsu, Mie 514-8507, Japan

2 Department of Electrical and Electronic Engineering, Mie University, Tsu, Mie, Japan 